\title{
Der BARF-Trend in der Hundeernährung - Eine Herausforderung für den Tierarzt?
}

\author{
Ingrid Vervuert, Cornelia Rückert
}

Das Füttern von sog. BARF-Rationen ist ein aktueller Ernährungstrend bei Hunden. Der Übersichtsartikel beschreibt das tierärztliche Spannungsfeld zwischen Motivation und Kenntnisstand der Hundebesitzer auf der einen Seite und den wesentlichen Nachteilen der BARFFütterung auf der anderen Seite. Bei vielen Hundebesitzern besteht der Wunsch nach einer „natürlichen und gesunden Fütterung“, wohingegen die Nachteile der BARF-Fütterung maßgeblich durch Imbalancen in der Nährstoffzufuhr und der Kontamination mit Salmonellen hauptsächlich durch rohes Geflügelfleisch gekennzeichnet sind.

\section{Einleitung}

Seit einigen Jahren ist das Füttern sog. BARF-Rationen bei Hundebesitzern besonders populär geworden. Die Abkürzung „BARF“ steht dabei für „,biologischartgerechtes rohes Futter" oder aber auch für „bones and raw foods“. Die wesentliche Motivation der Hundebesitzer für das Füttern von BARF-Rationen besteht in dem Wunsch nach einer „natürlichen, gesunden Fütterung“, gefolgt von gesundheitlichen Problemen des Hundes. Bei den gesundheitlichen Problemen werden in Besitzerumfragen vorrangig dermatologische oder gastrointestinale Erkrankungen ihres Hundes genannt [7, $16,17]$. Hervorzuheben ist dabei, dass $63 \%$ der Befragten mit der Umstellung auf eine BARF-Ration sehr zufrieden sind und mehr als $80 \%$ der zufriedenen Hundebesitzer auch in Zukunft barfen wollen ( Kasten) [17].

Im Unterschied zu den zahlreichen Besitzerumfragen gibt es kaum wissenschaftliche Untersuchungen, die die langfristigen Auswirkungen von BARFRationen zum Gegenstand hatten. In einer aktuellen Studie konnte gezeigt werden, dass sich die Zahngesundheit der Hunde nach Umstellung auf eine BARF-Ration aufgrund der Knochenfütterung innerhalb von 12 Wochen deutlich verbesserte [2]. Die Fell- und Kotqualität veränderten sich hingegen nicht wesentlich. Umgekehrt werden bei Rationskontrollen zahlreiche Abweichungen in der Nährstoffversorgung dargestellt ( Kasten): Häufig sind die Kalzium- (Ca) und Phosphor- (P) sowie die Spurenelementversorgung in den BARFRationen marginal. Die fettlöslichen Vitamine A und D können in Abhängigkeit von den Rationskomponenten sowohl sehr niedrig als auch extrem hoch sein $[7,10,13]$. In einer Untersuchung von Wendel et al. konnte auch gezeigt werden, dass in einer kommerziellen BARFRation für Welpen, die als „Alleinfutter“ deklariert war, nur 20\% der minimalen Empfehlung für die Kalziumzufuhr für Welpen enthalten war [18]. Rechtlich impliziert ein Alleinfutter, dass bei sachgemäßem Einsatz der gesamte Energieund Nährstoffbedarf des Tieres durch die ausschließliche Fütterung des Alleinfutters abgedeckt sein muss.

\section{Kenntnisse des Tierhalters?}

Die sog. „natürliche Fütterung“ orientiert sich am Beuteschema der wilden Vorfahren des Hundes.

BARF-Rationen bestehen im Wesentlichen aus ( $\triangleright$ Tab. 1) $[3,16]$ :

- rohem Fleisch, z.B. Rind, Geflügel, Pferd, Reh, Lamm

- Innereien, z. B. Herz, Lunge, Leber, Pansen

- Fisch

- Milchprodukten

- Gemüse

- Obst

- Pflanzenölen

- Knochen
Vor- und Nachteile von BARF-Rationen

\section{Vorteile}

- Kenntnis der Einzelkomponenten (Ausnahme: kommerzielle Produkte)

- längere Fresszeiten (Ausnahme: gewolfte Produkte)

- Zahngesundheit bei Knochenfütterung

- Reduktion der Kotmenge und verbesserte Kotkonsistenz

\section{Nachteile}

- Nährstoffimbalancen:

- Proteinüberversorgung

- Über- und Unterversorgung mit Mengen- und Spurenelementen

- Über- und Unterversorgung mit Vitaminen

- Übertragung von Krankheitserregern:

- Bakterien, z. B. Salmonellen

- Viren, z. B. Aujeszky

- Parasiten, z. B. Sarcocystis spp.

- Obstipationen durch Knochenfütterung

- Hyperthyreoidismus durch die Aufnahme von Schilddrüsengewebe bei der Schlundfütterung

- Aufnahme von antinutritiven Faktoren, z. B. Thiaminasen in rohem Fisch 
Tab. 1 Tierische Rationskomponenten in BARF-Rationen, die regelmäßig verwendet werden [16].

\begin{tabular}{|l|l|}
\hline Komponente & $\begin{array}{l}\text { Häufig- } \\
\text { keit (\%) }\end{array}$ \\
\hline Rindfleisch & 84 \\
\hline Geflügel & 72 \\
\hline Schlund & 57 \\
\hline Leber & 86 \\
\hline Herz & 82 \\
\hline Fisch (insbesondere Lachs) & 41 \\
\hline Knochen & 41 \\
\hline
\end{tabular}

Rund 26\% der Hundebesitzer füttern des Weiteren regelmäßig kleine Mengen an thermisch aufbereiteten Kohlenhydraten, z.B. Reis, Kartoffeln oder Nudeln [3]. Zusätzlich werden die Rationen teilweise mit weiteren Ergänzungen komplettiert, z.B. Eigelb, Nüssen oder Kräutern [16].

Die Futtermittel werden dabei im Wesentlichen von Metzgereien oder Supermärkten bezogen. Immerhin beziehen rund 30\% der Hundebesitzer die Futterkomponenten von Onlineshops.

Aus den verschiedenen Umfragen geht des Weiteren hervor, dass mehr als $90 \%$ der Hundebesitzer die BARF-Rationen als „sicher“ bezeichnen. Nähert man sich der Frage, woher die Tierhalter ihr Wissen über die korrekte Zusammenstellung der Ration entnehmen, so werden überwiegend das Internet oder populärwissenschaftliche Literatur genannt. Nur 14\% der befragten Hundehalter nutzen eine tierärztliche Fütterungsempfehlung [16].

\section{Herausforderung BARF- Rationen für den Tierarzt}

\section{Nährstoffimbalancen}

Aus Erhebungen zur typischen Energieund Nährstoffzusammensetzung von BARF-Rationen bei Hunden konnte gezeigt werden, dass 60-73\% der überprüften Rationen deutliche Abweichungen von den gültigen Energie- und Nährstoffempfehlungen aufwiesen [14].
Es konnte vor allem eine deutliche Unterversorgung mit folgenden Nährstoffen beobachtet werden ( $\triangleright$ Abb. 1) $[3,19]$ :

- Kalzium, inkl. inverses Ca/P-Verhältnis $<0,6: 1$

- Kupfer

- Zink

- Jod

- Vitamin A und Vitamin D

\section{konkret}

Um eine Ration adäquat einstellen zu können bzw. Imbalancen in der Nährstoffversorgung detektieren zu können, ist die Rationskalkulation das diagnostische Mittel der Wahl.

Bei der Rationsüberprüfung werden die mit der BARF-Ration aufgenommenen Nährstoffe mit den Bedarfsempfehlungen in Abhängigkeit von der Körpermasse des Hundes abgeglichen. Notwendige Korrekturen, z.B. die Ergänzung eines vitaminisierten Mineralfuttermit- 
tels, können berechnet werden. Die meisten veterinärmedizinischen Fakultäten, aber auch einige selbstständige Tierärzte bieten diese Beratung an.

\section{Hilft das BARF-Profil im Blut bei Nährstoffimbalancen?}

Zahlreiche Labore bieten ein sog. „BARFProfil“ an, wobei im Blut in der Regel das kleine Blutbild, Albumin, Kalzium, anorganisches Phosphat, Kupfer, Zink, Jod, Vitamin A und Vitamin D überprüft werden. Für die meisten der genannten Parameter besteht kein Zusammenhang zur aktuellen Fütterungspraxis, sodass man eine optimale Nährstoffversorgung nur sehr unzureichend anhand der genannten Blutparameter aufzeigen kann. Kalzium wird im Blut sehr eng homöostatisch reguliert, z.B. Kalzium-Freisetzung aus dem Knochen, sodass selbst bei einer dauerhaft marginalen Unterversorgung keine Veränderungen im Blut zu beobachten sind. Kupfer, Vitamin A und Vitamin D werden durch die Reserven in der Leber ebenfalls über einen langen Zeitraum im Blut unauffällig bleiben. Es gibt keinen Zusammenhang zur Zinkund Jodversorgung und den korrespondierenden Gehalten im Blut.

Mit gewissen Einschränkungen kann zumindest die Bestimmung des SerumRetinylpalmitats einen Hinweis auf die Vitamin-A-Versorgung des Hundes geben. Eine Erhöhung des SerumRetinylpalmitats kann bei einer sehr hohen Vitamin-A-Versorgung beobachtet werden, z. B. bei exzessiver Leberfütterung über mehrere Monate. Auch das 25-Hydroxycholecalciferol (Calcidiol) im Serum kann bei Werten, die unterhalb der empfohlenen Referenzwerte liegen, auf eine mehrmonatige Unterversorgung mit Vitamin D schließen lassen.

\section{Calcitriol im Serum bleibt selbst bei einer massiven Unterversorgung mit Vitamin D unverändert.}

Unter Berücksichtigung dieser Aspekte sind BARF-Profile eher irreführend, da sie bei unauffälligen Nährstoffkonzentrationen im Blut suggerieren, dass die BARF-Ration eine optimale Nährstoffversorgung bietet.

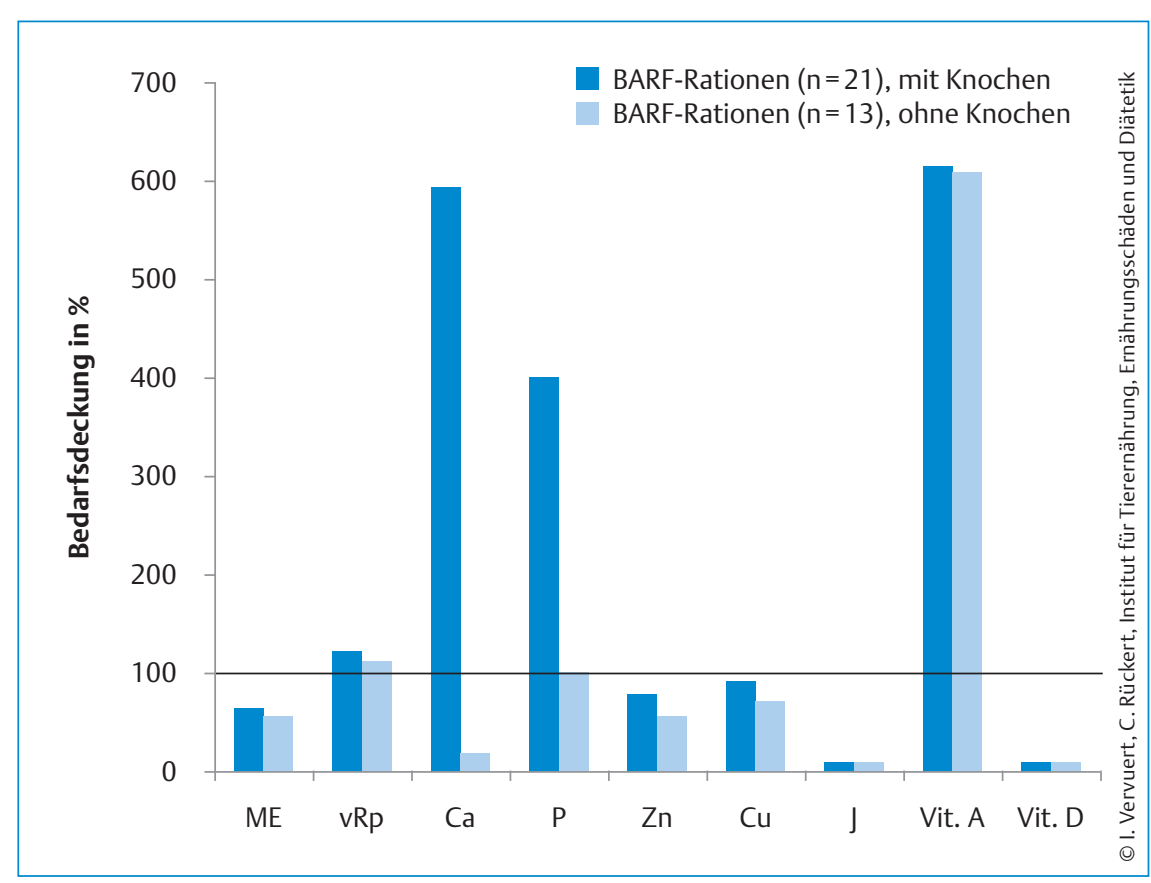

Abb. 1 Die durchschnittliche tägliche Versorgung mit Energie (ME), verdaulichem Rohprotein (vRp), Kalzium (Ca), Phosphor (P), Zink (Zn), Kupfer (Cu), Jod (J), Vitamin A (Vit. A) und Vitamin D (Vit. D) in zur Überprüfung eingesandten BARF-Futterplänen an das Institut für Tierernährung der Veterinärmedizinischen Fakultät Leipzig. Angaben des Bedarfs in \% nach National Research Council [14], schwarze Linie $100 \%$ = Bedarf.

\section{Salmonellen: Risiko rohes Fleisch}

Neben den deutlichen Imbalancen in der Nährstoffversorgung bei BARF-Rationen besteht auch ein erhebliches Hygienerisiko durch den Umgang und Verzehr von rohem Fleisch. Parasiten wie Bandwürmer, Viren wie das Aujeszky-Virus im rohen Schweinefleisch, aber auch Bakterien, insbesondere E. coli, Salmonellen oder Yersinien sind hierbei von Bedeutung.

In einer Untersuchung in Deutschland fielen von 15 gefrorenen Fertigrohfutterrationen 14 mit erhöhten aeroben Keimgehalten auf, sodass diese zumindest nicht mehr die Kriterien eines sicheren Lebensmittels erfüllten [18]. In Kanada wurden 166 kommerzielle Rohfutterabpackungen auf Salmonellen untersucht. Insgesamt wiesen $21 \%$ der untersuchten Proben Salmonellen auf, wobei $67 \%$ der Proben Hühnerfleisch enthielten [4]. Das Risiko, dass Hunde Salmonellen ausscheiden, wird bei Rationen mit einem hohen Anteil an Geflügel zwischen 23-44\% angegeben $[1,5,8,12]$. Hierbei steht weniger die Problematik im Vordergrund, dass die Hunde klinisch an einer Salmonellose erkranken, sondern die weitaus größere Gefahr besteht für die Gesundheit des Hundehalters. Hunde, die mit Salmonellen kontaminiertes Fleisch (insbesondere rohes Geflügelfleisch) aufnehmen, können über mehrere Wochen asymptomatisch Salmonellen ausscheiden. Eine Verteilung über den gesamten Haushalt mit der Möglichkeit der Infektion von Menschen kann nicht ausgeschlossen werden. Lefebvre et al. [11] fordern deshalb, dass Hunde, die in Kontakt mit immungeschwächten Personen stehen, nicht mit BARF-Rationen gefüttert werden sollten. In einer aktuellen Studie konnte darüber hinaus die Kontamination mit E. coli in BARF-Rationen nachgewiesen werden, wobei sich $23 \%$ der positiven Proben als Antibiotika-resistente E.-coliStämme erweisen [15].

\section{Was ist sonst noch zu beachten?}

\section{Fütterung von Schlund}

Bei BARF-Rationen werden häufig Kopfund Kehlfleisch inkl. Trachea gefüttert. Diese Futtermittel können Schilddrüsen- 
Tab. 2 Plasma-T4-Aktivitäten bei 2 Hunden mit nutritivem Hyperthyreoidismus bei Fütterung einer BARF-Ration bzw. Wechsel auf eine kommerzielle Ration (nicht näher spezifiziert) [9].

\begin{tabular}{|llll|}
\hline Hund & Ration & $\begin{array}{l}\text { Tag nach } \\
\text { Futter- } \\
\text { wechsel }\end{array}$ & $\begin{array}{l}\text { Plasma-T4- } \\
\text { Spiegel } \\
(\mathbf{m m o l} / \mathbf{l})^{1}\end{array}$ \\
\hline Fall 1 & BARF & 0 & 84,8 \\
\hline & kommerzielle Ration & 36 & 15,4 \\
\hline Fall 2 & BARF & 68 & 14,1 \\
\hline & kommerzielle Ration & 25 & 25,7 \\
\hline & & 0 & 167,1 \\
\hline
\end{tabular}

1 Referenzbereich von Plasma-T4: 19,3-51,4 mmol/l
Tab. 3 Antinutritive Faktoren in verschiedenen Futtermitteln.

\begin{tabular}{|c|c|c|}
\hline $\begin{array}{l}\text { Futter- } \\
\text { mittel }\end{array}$ & $\begin{array}{l}\text { antinutritiver } \\
\text { Faktor }\end{array}$ & Konsequenz \\
\hline Eier & $\begin{array}{l}\text { Avidin } \\
\text { (= Anti-Biotin) }\end{array}$ & $\begin{array}{l}\text { Hemmung der Biotinabsorption } \\
\text { aus dem Darm } \rightarrow \text { Hautverände- } \\
\text { rungen }\end{array}$ \\
\hline Fisch & Thiaminasen & $\begin{array}{l}\text { Abbau von Thiamin } \rightarrow \text { Paralyse, } \\
\text { Enzephalopathien }\end{array}$ \\
\hline Bohnen & Lektine & $\begin{array}{l}\text { Permeabilitätsstörungen, } \\
\text { insbesondere Darmschleimhaut } \rightarrow \\
\text { blutige Durchfälle }\end{array}$ \\
\hline $\begin{array}{l}\text { Bohnen, } \\
\text { Maniok }\end{array}$ & $\begin{array}{l}\text { zyanogene } \\
\text { Glykoside }\end{array}$ & $\begin{array}{l}\text { Blausäurevergiftung } \rightarrow \text { Salivation, } \\
\text { Dyspnoe, Taumeln, Festliegen, } \\
\text { Konvulsionen, Krämpfe und } \\
\text { Lähmung }\end{array}$ \\
\hline
\end{tabular}

gewebe enthalten, sodass es zu einem Hyperthyreoidismus aufgrund der exzessiven Aufnahme von Schilddrüsenhormonen kommen kann [9]. Das Schilddrüsenhormon T4 ist sehr gut geeignet, um einen nutritiven Hyperthyreoidismus detektieren zu können. Bei den meisten Hunden kommt es nach Diagnosestellung und dem Wechsel von einer BARF-Ration auf eine kommerzielle Diät zu einer Normalisierung der Hormonaktivitäten ( $\bullet$ Tab. 2). Auch die klinischen Symptome, falls vorhanden, sind bei den meisten Hunden reversibel.

\section{Umgang mit antinutritiven Faktoren?}

Neben der Auswahl von potenziell toxischen Futtermitteln wie Trauben, Avocados, Zwiebeln oder Knoblauch bergen BARF-Rationen das Risiko, dass Futtermittel angeboten werden, die sich nicht zur rohen Fütterung eignen. In > Tab. 3 werden Futtermittel mit antinutritiven Faktoren vorgestellt, die erst durch das Erhitzen deaktiviert werden.

\section{Fazit}

Seit einigen Jahren ist das Füttern sog. BARF-Rationen bei Hundebesitzern besonders populär geworden. Auf der einen Seite stehen bei der Fütterung der BARFRationen der Wunsch des Hundebesitzers nach einer „natürlichen, gesunden Fütterung“ oder gesundheitliche Probleme, z.B. dermatologische Erkrankungen des Hundes, im Vordergrund. Auf der an- deren Seite fallen viele BARF-Rationen durch Imbalancen in der Nährstoffzufuhr auf. Des Weiteren ist die Rohfütterung, insbesondere von Geflügelfleisch, mit einem erheblichen Risiko der Salmonellenausscheidung durch den Hund behaftet. Tierärzte sollten den Trend der Fütterung von BARF-Rationen stärker begleiten und den Tierhalter über Vor- und Nachteile der Rohfütterung aufklären. Die Rationsüberprüfung ist dabei als tierärztliche Aufgabe zu verstehen, wohingegen das BARF-Profil irreführend in Bezug auf die Beurteilung einer adäquaten Nährstoffversorgung ist.

\section{Online zu finden unter}

http://dx.doi.org/10.1055/s-0043-101858

\section{Literatur}

1 Ansel AK, Flekna G, Stessl B, Iben C. Salmonella in food and in faeces of dogs fed raw food (BARF). Berlin: Proceedings ESVCN-Kongress; 2016: 130

2 Bieri M, Liesegang A. Effect of BARF-feeding on faecal consistency, coat quality, dental health and blood values of dogs. Berlin: Proceedings ESVCN-Kongress; 2016: 48

3 Dillitzer N, Becker N, Kienzle E. Intake of minerals, trace elements and vitamins in bone and raw food rations in adult dogs. $\mathrm{Br}$ J Nutr 2011; 106: 553-556

4 Finley R, Reid-Smith R, Ribble C et al. The occurrence and antimicrobial susceptibility of salmonellae isolated from commercially available canine raw food diets in three
Canadian cities. Zoonoses Public Health 2008; 55: 462-469

5 Finley R, Ribble C, Aramini ] et al. The risk of salmonellae shedding by dogs fed Salmonella-contaminated commercial raw food diets. Can Vet J 2007; 48 (81): 69-75

6 Handl S. Der „BARF“-Trend - Vorteile, Nachteile und Risiken. Vet Focus 2014; 24 (3): $16-23$

7 Handl S, Zimmermann S, Iben C. Reasons for dog owners to choose raw diets („BARF“) and nutritional adequacy of raw diet recipes fed to dogs in Austria and Germany. Poland: Proceedings ESVCN congress; 2012: 124

8 Joffe DJ, Schlesinger DP. Preliminary assessment of the risk of Salmonella infection in dogs fed raw chicken diets. Can Vet J 2002; 43 (6): 441-442

9 Köhler B, Stengel C, Neiger R. Dietary hyperthyroidism in dogs. J Small Anim Practice 2012; 53 (3): 182-184

Die Literatur 10-19 ist in der Online-Version unter www.Thieme-connect.de/products einsehbar.

PD Dr. med. vet. Ingrid Vervuert

Dr. med. vet. Cornelia Rückert

Universität Leipzig

Veterinärmedizinische Fakultät

Institut für Tierernährung, Ernährungsschäden und Diätetik

An den Tierkliniken 9

04103 Leipzig

ingrid.vervuert@vetmed.uni-leipzig.de 
10 Kölle P, Schmidt M. BARF (biologisch artgerechte Rohfütterung) als Ernährungsform bei Hunden. Tierarztl Prax 2015; 43 (K): 409-419

11 Lefebvre SL, Reid-Smith R, Boerlin P et al. Evaluation of the risks of shedding Salmonellae and other potential pathogens by therapy dogs fed raw diets in Ontario and Alberta. Zoonoses Public Health 2008; 55 : $470-480$

12 Leonard EK, Pearl DL, Finley RL et al. Evaluation of pet-related management factors and the risk of Salmonella spp. carriage in pet dogs from volunteer households in Ontario (2005-2006). Zoonoses Public Health 2011; 58 (2): 140-149

13 Mack JK, Kienzle E. Fehlversorgungen in „BARF“-Futterplänen für einen Wurf Berner-Sennenhund-Welpen. Tierarztl Prax 2016; 44 (K): 341-347

14 National Research Council (NRC). Nutrient Requirements of Dogs and Cats. Washington DC, USA: National Academic Press; 2006

15 Nilsson $\mathrm{O}$. Hygiene quality and presence of ESBL-producing Escherichia coli in raw food diets for dogs. Infect Ecol Epidemiol 2015; 5: 28758

16 Ricci R, Morelli G, Fusi E, Danesi A, Bastianello S, Catellani P, Diez M. Attitudes of dog owners using BARF in Italy: a survey. Berlin: Proceedings ESVCN-Kongress; 2016: 131

17 Virtanen J, Sjörgen N, Roine J, Uusitalo L, Hielm-Björkman A. exploratory report: 632 shared experiences from dog owners changing their dogs' food to a raw food (BARF) diet. Berlin: Proceedings ESVCN-Kongress; 2016: 132

18 Wendel F, Kienzle E, Bohnke R, Dobenecker B. Microbiological contamination and inappropriate composition of BARF-food. Poland: Proceedings ESVCN congress; 2012: 67

19 Zimmermann S. Umfrage zum Thema Rohfütterung „BARF“ unter Hundebesitzern in Österreich und Deutschland und rechnerische Überprüfung von BARF-Rationen [Diplomarbeit]. Wien: Veterinärmedizinische Universität Wien; 2013 